\title{
Domain structure formation by local switching in the ion sliced lithium niobate thin films
}

\author{
Cite as: Appl. Phys. Lett. 116, 152904 (2020); doi: 10.1063/5.0005969 \\ Submitted: 27 February 2020 - Accepted: 28 March 2020 • \\ Published Online: 13 April 2020
}

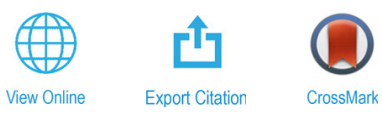

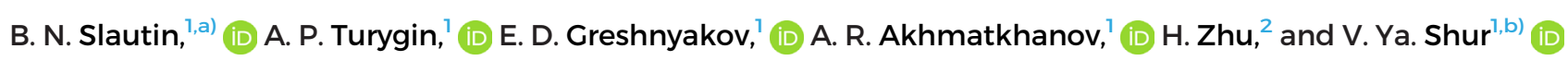

\author{
AFFILIATIONS \\ ${ }^{1}$ School of Natural Sciences and Mathematics, Ural Federal University, 620000 Ekaterinburg, Russia \\ 2Jinan Jingzheng Electronics Co. Ltd., Jinan 250101, China

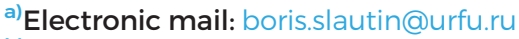 \\ b) Author to whom correspondence should be addressed: vladimir.shur@urfu.ru
}

\begin{abstract}
The creation of the periodical domain patterns with a submicron period in lithium niobate on insulator (LNOI) wafers is a key problem for nonlinear-optical applications, including second harmonic generation, backscattering optical parametric oscillator, etc. We have experimentally studied the domain formation and evolution during local polarization reversal in Z+ LNOI wafers with a metal bottom electrode. It has been shown that domain growth occurs by the formation of the spikes at the charged domain wall (CDW). The complicated shape of isolated domains with a jagged CDW has been revealed. The obtained weak domain-domain interaction has been attributed to effective bulk screening by charge injection. The revealed dependence of the domain sizes on humidity caused by the adsorbed water layer should be taken into account during periodical poling.
\end{abstract}

Published under license by AIP Publishing. https://doi.org/10.1063/5.0005969

Lithium niobate $\mathrm{LiNbO}_{3}$ (LN) on insulator wafers (LNOI) consisting of a submicrometer-thick $\mathrm{LN}$ film bonded to the $\mathrm{SiO}_{2}$ layer deposited on the thick LN substrates was first produced by ion cutting in 2004. The high contrast of refractive index between the LN film and the $\mathrm{SiO}_{2}$ layer allows realizing integrated optical devices based on photonic wires and bandgap structures. ${ }^{2,3}$ Today, LNOI is one of the most promising materials for various nonlinear-optical applications, such as high-effective low-loss waveguides, ${ }^{1,4,5}$ electro-optical modulators, ${ }^{6}$ and wavelength convertors.

Creation of the stripe periodical domain structure in LN crystals (periodical poling, PPLN) allows using the quasi-phase matching effect for the improvement of various optical devices. ${ }^{9,10}$ The precise domain structures increase essentially the efficiency of the nonlinear frequency convertors by second harmonic and difference frequency generation. ${ }^{9,10}$ The backward quasi-phase matching (B-QPM) with counter-propagating waves needs PPLN with submicrometer periods. B-QPM allows creating backwardgenerated optical parametric oscillators without an external cavity, which has an internal feedback for parametric light generation. ${ }^{11}$ B-QPM was also proposed for slow photon generation ${ }^{12}$ and pulse shaping. ${ }^{13}$

Local polarization reversal by scanning probe microscopy (SPM) is promising for periodical poling with a submicrometer period. ${ }^{14}$ The
PPLN with a period of about $300 \mathrm{~nm}$ has been created by local switching on the small areas of the LNOI wafers. ${ }^{15}$

The domain growth and interaction during local switching of LNOI wafers have been studied by piezoresponse force microscopy (PFM). ${ }^{15-20}$ Formation of the stable isolated domains with charged domain walls (CDWs) and the absence of domain interaction have been found in LNOI wafers with a metal electrode under the LN film. ${ }^{15-17}$ The thermal stability of the created domains after the preheat treatment has been shown recently. ${ }^{20}$ The domain imaging at the cross section of LNOI by scanning transmission electron microscopy revealed the much stronger domain wall inclination, ${ }^{21}$ compared to bulk LN crystals. $^{22,23}$ The further decrease in PPLN periods and increase in the domain pattern stability need the deeper study of the domain growth during local switching in LNOI wafers.

The influence of relative humidity $(\mathrm{RH})$ on the switching kinetics during local switching has attracted great attention in recent years and has been studied in congruent ${ }^{24}$ and $\mathrm{MgO}$ doped ${ }^{25,26} \mathrm{LN}$ crystals, but never in LNOI wafers. It has been demonstrated that the domain growth depends on the electric field produced by the SPM tip with a meniscus of the adsorbed water. ${ }^{24,27,28}$ Moreover, the water layer influences the domain kinetics. ${ }^{28-30}$ This problem is tightly related to the emerging field of surface electrochemistry phenomena during local switching in ferroelectrics. ${ }^{31,32}$ 
In this article, we have studied the growth and interaction of isolated domains during local switching using domain imaging by PFM and confocal Raman microscopy (CRM). The main mechanisms of domain evolution and influence of the humidity have been proposed.

We have studied LNOI wafers with a metal bottom electrode with the following configuration: $\mathrm{LN}$ film $(700 \mathrm{~nm}) / \mathrm{Cr}(30 \mathrm{~nm}) / \mathrm{Pt}$ $(100 \mathrm{~nm}) / \mathrm{Cr}(10 \mathrm{~nm}) / \mathrm{SiO}_{2}(2 \mu \mathrm{m}) / \mathrm{LN}$ substrate $(500 \mu \mathrm{m})$. The surface roughness was below $1 \mathrm{~nm}$.

Switching and domain imaging were carried out by means of a scanning probe microscope NTEGRA Aura (NT-MDT, Russia). The rectangular DC voltage pulses with various amplitudes and durations were applied by the conductive tip, whereas the solid bottom electrode was grounded. The cantilever was raised above the surface before the pulse termination to avoid spontaneous backswitching. The pulse voltage ranged from 40 to $120 \mathrm{~V}$ and the pulse duration from $10 \mathrm{~ms}$ to 100 s. Domain imaging at the surface was performed by single frequency PFM with an amplitude of $\mathrm{AC}$ voltage of $6 \mathrm{~V}$ and a frequency of $21 \mathrm{kHz}$. Conductive $\mathrm{W}_{2} \mathrm{C}$ coated cantilevers $\mathrm{HA} \mathrm{HR} /$ W2C+ (ScanSens GmbH, Hamburg, Germany) with a spring constant of about $17 \mathrm{~N} / \mathrm{m}$ were used. The spatial resolution of PFM domain imaging was about $20 \mathrm{~nm}$. The domain wall conductivity was measured by conductive atomic force microscopy (CAFM). All experiments were realized in nitrogen with $\mathrm{RH}$ controlled by the self-made setup and measured using a SPM humidity sensor.

Domain imaging in the film bulk was realized by CRM using an Alpha 300 AR (WiTec GmbH, Ulm, Germany) with a $488 \mathrm{~nm}$ laser. The laser beam was focused by a $100 \times$ objective with $0.75 \mathrm{NA}$, and the scattered light was collected by the same objective in the backscattering geometry. The light decomposition to the spectrum was done by 1800 grids $/ \mathrm{mm}$ grating, providing the spectral resolution of $1.22 \mathrm{~cm}^{-1}$. Scanning was carried out by the high-precision piezoelectric stage. The spatial resolution was about $300 \mathrm{~nm}$.

We have studied the influence of RH on the domain growth during local switching. The irregular shaped domain size has been characterized by effective diameter $D=2 \sqrt{A / \pi}$, where $A$ is a domain area in the phase PFM image. The diameters of five domains created at the same conditions have been averaged. The linear dependence of $D$ on the pulse amplitude [Fig. 1(a)] and the logarithmic dependence on the pulse duration [Fig. 1(b)] were observed for all $\mathrm{RH}$ values. Both dependences are typical for local switching in bulk LN crystals. ${ }^{33,34}$ The domain size increases nonlinearly with increasing RH [Figs. 1(c) and 1(d)]. At high RH values, after the application of the field pulses with a large amplitude or after duration $D$ reached several micrometers, an increase in the size with $\mathrm{RH}$ in large domains has been observed for thin Mg:LN crystals ${ }^{26}$ and thin lead zirconate titanate (PZT) films. ${ }^{28}$

The influence of $\mathrm{RH}$ on the domain growth is qualitatively different for small and large domains. For small domains appearing at low voltages, the water layer decelerates the domain wall motion due to delocalization of the field under the tip. ${ }^{24,25}$ The obtained increase in the domain growth velocity with humidity for large domains [Figs. 1(c) and 1(d)] can be attributed to (1) the water meniscus at the tip apex ${ }^{27,35}$ and (2) the low-conductive water layer over the surface. ${ }^{27,30}$ The area of the contact between the tip and the surface increases by the meniscus, playing the role of a top electrode. Moreover, the water layer can be considered as an effective electrode. The domain wall velocity is controlled by the charge flow (switching current), which is necessary for compensation of the changes in bound charge on the
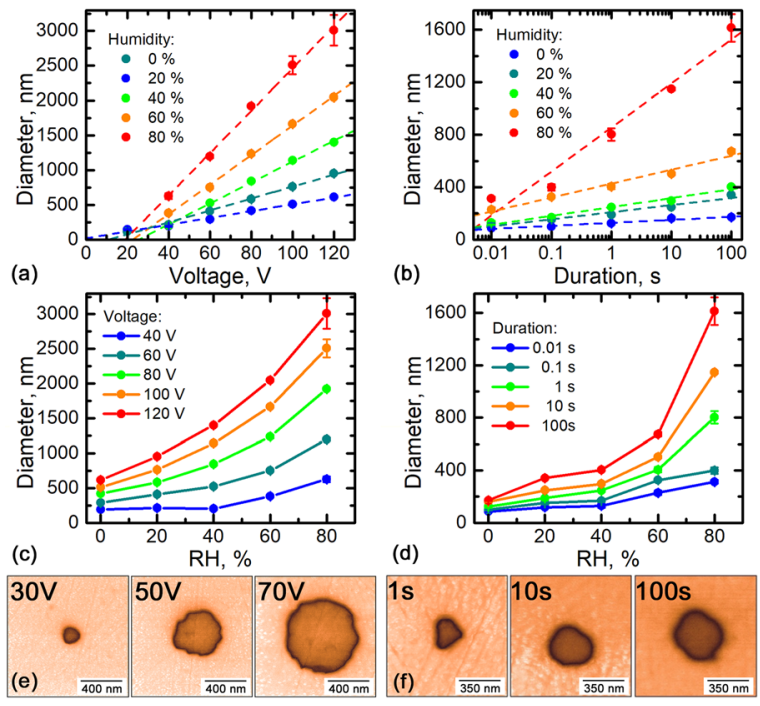

FIG. 1. Dependence of the effective domain size at different $\mathrm{RH}$ values on (a) and (c) pulse amplitude (pulse duration $1 \mathrm{~s}$ ) and (b) and (d) pulse duration (voltage $40 \mathrm{~V}$ ). The typical domain shapes for various (e) pulse amplitudes (duration $5 \mathrm{~s}$ ) and (f) pulse durations (voltage $40 \mathrm{~V}$ ).

polar surface. The increase in the water layer depth with $\mathrm{RH}$ leads to the decrease in its resistivity. Therefore, the RH increase leads to the acceleration of the domain wall motion. The influence of the electrochemical phenomena and water splitting, which can promote or hinder the domain growth, was neglected. ${ }^{3}$

The typical domain shapes for various pulse amplitudes [Fig. $1(\mathrm{e})$ ] and durations [Fig. $1(\mathrm{f})$ ] at $\mathrm{RH}=20 \%$ are presented. It is seen that the growing domains transformed from the rounded triangles to the approximately circular ones with the wavy walls for D > $300 \mathrm{~nm}$.

In addition, we used CRM for the domain wall imaging, which has been used successfully for bulk LN crystals. ${ }^{37}$ The domain wall contrast in CRM images was obtained by measuring the local values of the effective shift of the center mass of $\mathrm{E}\left(\mathrm{TO}_{8}\right)$ and $\mathrm{A}_{1}\left(\mathrm{LO}_{4}\right)$ Raman lines. The contrast of the charged domain walls is essentially higher than that of the neutral walls. It is necessary to point out that the film thickness in LNOI is above the spatial resolution of CRM $(300 \mathrm{~nm})$. For the rectangular domain created by scanning the area with the application of $70 \mathrm{~V}$ DC voltage [Fig. 2(a)], the CRM contrast was obtained over the whole switched area [Fig. 2(b)]. A similar contrast was obtained in the crystals of relaxor ferroelectric strontium barium niobate (SBN) with nonthrough nanodomains with CDWs. ${ }^{38}$ This fact can be attributed to the existence of the domains with CDWs. The measured electric current ( $15 \mathrm{pA}$ for $5 \mathrm{~V} \mathrm{DC}$ voltage) at the domain walls [Figs. 2(c) and 2(d)] can be attributed to the head-to-head CDW with intrinsic electron conductivity. ${ }^{39,40}$

CRM imaging of the created isolated domains [Fig. 2(e)] allowed us to reveal that the nanoscale vertical shift of the beam focus by the high-precision piezoelectric stage from the surface into the depth led to the significant change in the domain image [Figs. 2(f)-2(h)]. This fact can be used for qualitative characterization of the domain wall shape in the LN thin film. The decrease in the area with CRM contrast has been attributed to the decrease in the CDW density. 


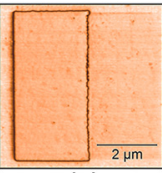

(a)

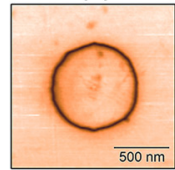

(e)

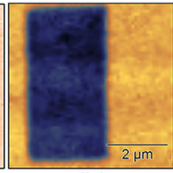

(b)

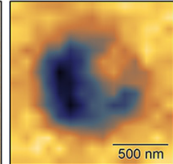

(f)

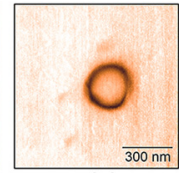

(c)

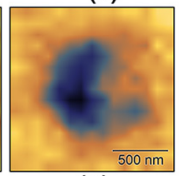

(g)

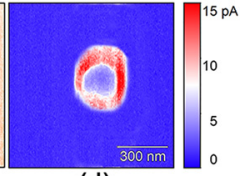

(d)

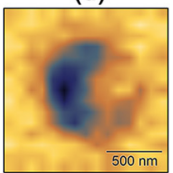

(h)
FIG. 2. The switched domains: (a), (c), and (e) PFM amplitudes, (d) local current, CRM contrast (b) rectangular domain; $(\mathrm{f}),(\mathrm{g})$, and $(\mathrm{h})$ isolated domain: (f) at the LN surface and at different depths: (g) $230 \mathrm{~nm}$ and (h) $460 \mathrm{~nm}$.

The domain shape in the bulk and mechanism of domain growth were studied by repeated gradual removal of the isolated domains by continuous scanning with DC voltage below the threshold $(-10 \mathrm{~V})$ and PFM imaging. ${ }^{41}$ Two scenarios of the domain change have been separated. First, scanning leads to a decrease in the domain size followed by the appearance of the quasiregular nanodomain chain at $100 \mathrm{~nm}$ from the domain wall [Figs. 3(a), 3(c), 3(e), 3(g)]. Second, the scanning leads to the appearance of the star domain with nanodomains between the rays, which are divided into isolated nanodomains after several scans [Figs. 3(b), 3(d), 3(f), and 3(h)].

The interaction of the small isolated domains was studied in the domain arrays with various periods, and the same diameter (about $130 \mathrm{~nm}$ ) switched in dry nitrogen (Fig. 4). It was shown that the domain size and shape did not change with the decreasing period. In spite of the CDW, a noticeable interaction was not observed up to merging. This fact contradicts to the pronounced domain-domain interaction obtained in the bulk LN crystals. ${ }^{42}$ The difference can be attributed to the effective bulk screening of the depolarization field by carrier injection in LN thin films. ${ }^{4.3}$

We can reconstruct qualitatively, by analysis of CRM images, the shape of a domain in the bulk of the film with the CDW revealed by CAFM and STEM $^{21}$ [Figs. 2(f)-2(h)]. It was mentioned above that CRM contrast was due to the CDW. ${ }^{37}$ Thus, the single isolated domain is solid only at the surface, having conical spikes in the bulk (Fig. 5). The obtained contrast variation can be attributed to the existence of the jagged CDW with different sizes of the conical spikes.

The observed shape of the switched domain at the surface after partial backswitching (Fig. 3) can be attributed to two growth mechanisms: (1) the correlated nucleation (discrete switching) with the formation of the nanodomains in front of the moving domain wall [Fig. 5(b)] and (2) formation and forward growth of the conical nanodomains (spikes) at the CDW [Fig. 5(c)]. The first mechanism was discovered by us in lead germanate $\mathrm{Pb}_{5} \mathrm{Ge}_{3} \mathrm{O}_{11}{ }^{44,45}$ and studied in bulk LN crystals. ${ }^{9,10}$ According to the second mechanism, the forward growth of isolated needle-like domains in the polar direction is a result of step generation and kink growth. ${ }^{22}$ The growth rate is determined by the local electric field, which is the sum of external, depolarization, and screening fields. The tip of the needle-like domain cannot reach the bottom electrode due to the existence of an intrinsic dielectric gap, which makes impossible the complete screening by current in the external circuit. ${ }^{22}$ The field produced by the terminated tip leads to
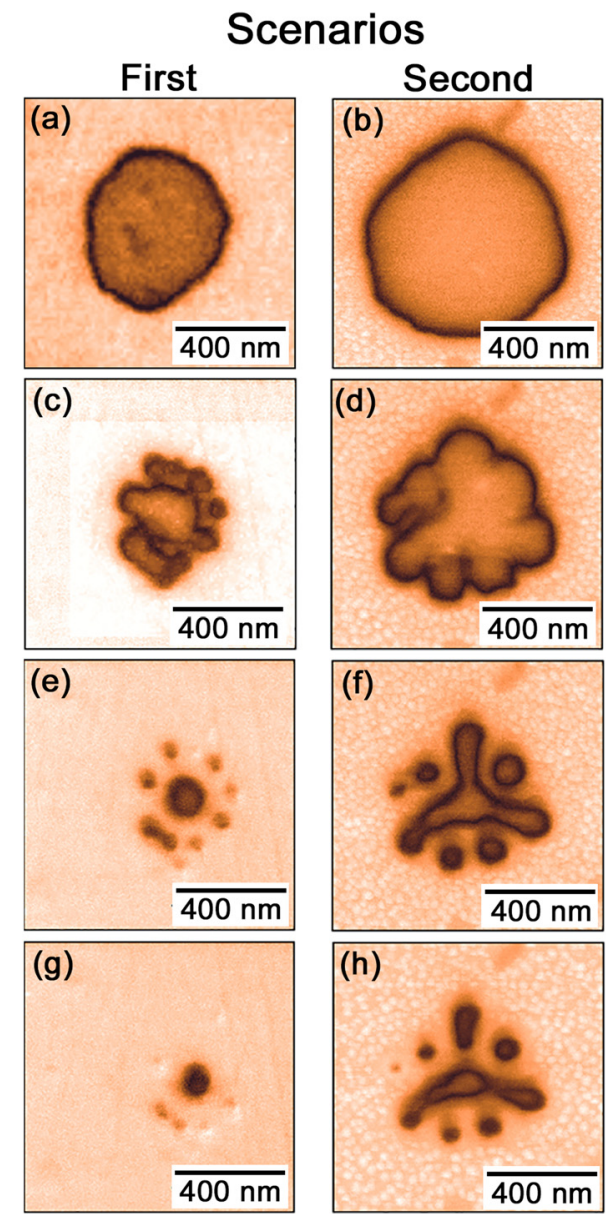

FIG. 3. Scenarios of the domain gradual removal as a result of scanning with a DC voltage of $-10 \mathrm{~V}$ : (a) and (b) initial states; after scans: (c) and (d) 1; (e) and (f) 4; and $(\mathrm{g})$ and $(\mathrm{h}) 6$.

deceleration and termination of the kink motion. The increase in the kink concentration results in the formation of the CDW abnormally tilted from the polar direction. The high local value of the depolarization field leads to the generation of the additional spike at the wall,

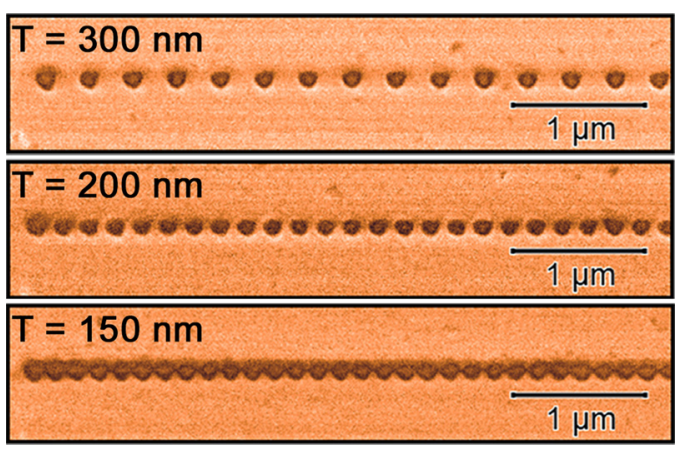

FIG. 4. PFM images of nanodomain arrays switched in dry nitrogen. 


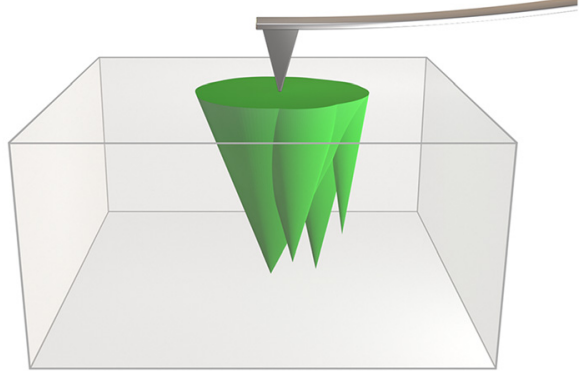

(a)

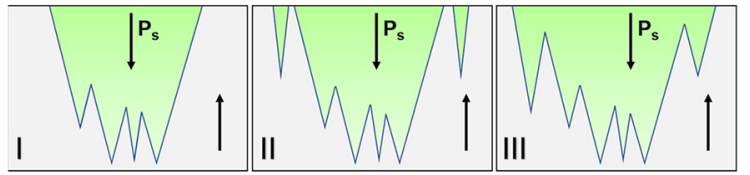

(b)

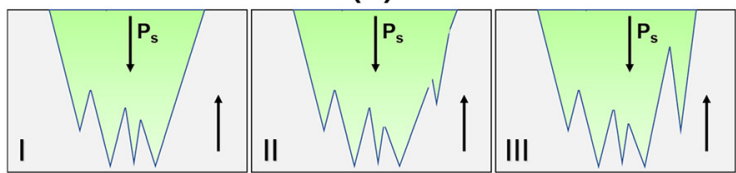

(c)

FIG. 5. Schematic view of (a) isolated domain; domain growth by: (b) discrete switching and (c) spike generation at the CDW.

which grows and stops at the gap [Fig. 5(c)-(II)]. ${ }^{22}$ Formation and growth of the spike reduce the average CDW tilt by the partial backward switching [Fig. 5(c)-(III)]. ${ }^{23}$ This cyclic process provides formation of the jagged CDW. The distance between the spikes is controlled by the applied field value.

Nanodomain structures observed in the LNOI as a result of partial removal of the isolated domain were associated earlier with the correlated nucleation. ${ }^{41}$ At the same time, the nanodomains in front of a domain wall appeared only as a result of partial removal and never have been imaged in LNOI just after the domain switching. This fact supports the second mechanism.

In conclusion, the local switching was studied in the LNOI wafers. It was shown that the domains had a complicated 3D shape with jagged domain walls. The process of the domain growth occurred by the generation of the spikes at the CDW. The observed extremely weak interaction between domain walls is a key property for creation of the high quality periodical domain structures with submicron periods. It was shown that the domain size strongly depended on the humidity. The observed acceleration of the wall motion velocity for large domains with $\mathrm{RH}$ caused by the influence of the low-conductive water layer at the surface should be taken into account during periodical poling.

The equipment of the Ural Center for Shared Use "Modern Nanotechnology" Ural Federal University was used. The authors thank E. A. Linker for the help in processing of the 3D model. This research was made possible by RFBR (Grant Nos. 19-32-90052 and 20-42-660025).

The data that support the findings of this study are available from the corresponding author upon reasonable request.

\section{REFERENCES}

${ }^{\mathbf{1}}$ P. Rabiei and P. Gunter, Appl. Phys. Lett. 85, 4603 (2004).

${ }^{2}$ A. Boes, B. Corcoran, L. Chang, J. Bowers, and A. Mitchell, Laser Photonics Rev. 12, 1700256 (2018).

${ }^{3}$ G. Poberaj, H. Hu, W. Sohler, and P. Günter, Laser Photonics Rev. 6, 488 (2012).

${ }^{4}$ H. Han, L. Cai, and H. Hu, Opt. Mater. 42, 47 (2015).

${ }^{5}$ M. F. Volk, S. Suntsov, C. E. Rüter, and D. Kip, Opt. Express 24, 1386 (2016).

${ }^{6}$ C. Wang, M. Zhang, B. Stern, M. Lipson, and M. Lončar, Opt. Express 26, 1547 (2018).

${ }^{7}$ L. Chang, Y. Li, N. Volet, L. Wang, J. Peters, and J. E. Bowers, Optica 3, 531 (2016).

${ }^{8}$ C. Wang, X. Xiong, N. Andrade, V. Venkataraman, X.-F. Ren, G.-C. Guo, and M. Lončar, Opt. Express 25, 6963 (2017).

${ }^{9}$ V. Ya. Shur, A. R. Akhmatkhanov, and I. S. Baturin, Appl. Phys. Rev. 2, 040604 (2015).

${ }^{10}$ A. I. Lobov, V. Ya. Shur, D. K. Kuznetsov, S. A. Negashev, D. V. Pelegov, E. I. Shishkin, and P. S. Zelenovskiy, Ferroelectrics 373, 99 (2008).

${ }^{11}$ S. E. Harris, Appl. Phys. Lett. 9, 114 (1966).

12. B. Khurgin, Phys. Rev. A 72, 023810 (2005).

${ }^{13}$ M. Conforti, C. de Angelis, U. K. Sapaev, and G. Assanto, Opt. Express 16, 2115 (2008).

${ }^{14}$ S. Moscovich, A. Arie, R. Urneski, A. Agronin, G. Rosenman, and Y. Rosenwaks, Opt. Express 12, 2236 (2004).

${ }^{15}$ T. Volk, R. Gainutdinov, and H. Zhang, Crystals 7, 137 (2017).

${ }^{16}$ R. V. Gainutdinov, T. R. Volk, and H. H. Zhang, Appl. Phys. Lett. 107, 162903 (2015).

17T. R. Volk, R. V. Gainutdinov, and H. H. Zhang, Appl. Phys. Lett. 110, 132905 (2017).

${ }^{18}$ J. Jiang, X. J. Meng, D. Q. Geng, and A. Q. Jiang, J. Appl. Phys. 117, 104101 (2015).

${ }^{19}$ G. Shao, Y. Bai, G. Cui, C. Li, X. Qiu, D. Geng, D. Wu, and Y. Lu, AIP Adv. 6, 075011 (2016).

${ }^{20}$ Y. Jiao, Z. Shao, S. Li, X. Wang, F. Bo, J. Xu, and G. Zhang, Crystals 10, 74 (2020).

${ }^{21}$ H. Lu, Y. Tan, J. P. V. McConville, Z. Ahmadi, B. Wang, M. Conroy, K. Moore, U. Bangert, J. E. Shield, L.-Q. Chen, J. M. Gregg, and A. Gruverman, Adv. Mater. 31, 1902890 (2019).

${ }^{22}$ V. Ya. Shur, E. L. Rumyantsev, E. V. Nikolaeva, and E. I. Shishkin, Appl. Phys, Lett. 77, 3636 (2000).

${ }^{23}$ A. A. Esin, A. R. Akhmatkhanov, and V. Ya. Shur, Appl. Phys. Lett. 114, 092901 (2019).

${ }^{24}$ A. V. Ievlev, A. N. Morozovska, V. Ya. Shur, and S. V. Kalinin, Appl. Phys. Lett. 104, 092908 (2014).

${ }^{25}$ V. Ya. Shur, A. V. Ievlev, E. V. Nikolaeva, E. I. Shishkin, and M. M. Neradovskiy, J. Appl. Phys. 110, 052017 (2011).

${ }^{26}$ S. M. Neumayer, E. Strelcov, M. Manzo, K. Gallo, I. I. Kravchenko, A. L. Kholkin, S. V. Kalinin, and B. J. Rodriguez, J. Appl. Phys. 118, 244103 (2015).

${ }^{27}$ D. Dahan, M. Molotskii, G. Rosenman, and Y. Rosenwaks, Appl. Phys. Lett. 89, 152902 (2006).

${ }^{28}$ C. Blaser and P. Paruch, New J. Phys. 17, 013002 (2015).

${ }^{29}$ S. V. Kalinin, Y. Kim, D. D. Fong, and A. N. Morozovska, Rep. Prog. Phys. 81, 036502 (2018).

${ }^{30}$ A. Brugère, S. Gidon, and B. Gautier, J. Appl. Phys. 110, 052016 (2011).

${ }^{31}$ A. V. Ievlev, C. C. Brown, J. C. Agar, G. A. Velarde, L. W. Martin, A. Belianinov, P. Maksymovych, S. V. Kalinin, and O. S. Ovchinnikova, ACS Appl. Mater. Interfaces 10, 38217 (2018).

${ }^{32}$ S. M. Neumayer, A. V. Ievlev, L. Collins, R. Vasudevan, M. A. Baghban, O. Ovchinnikova, S. Jesse, K. Gallo, B. J. Rodriguez, and S. V. Kalinin, ACS Appl. Mater. Interfaces 10, 29153 (2018).

${ }^{33}$ K. Terabe, M. Nakamura, S. Takekawa, K. Kitamura, S. Higuchi, Y. Gotoh, and Y. Cho, Appl. Phys. Lett. 82, 433 (2003).

${ }^{34}$ B. J. Rodriguez, R. J. Nemanich, A. Kingon, A. Gruverman, S. V. Kalinin, K. Terabe, X. Y. Liu, and K. Kitamura, Appl. Phys. Lett. 86, 012906 (2005).

${ }^{35}$ B. L. Weeks, M. W. Vaughn, and J. J. Deyoreo, Langmuir 21, 8096 (2005). 
${ }^{36}$ S. V. Kalinin, S. Jesse, A. Tselev, A. P. Baddorf, and N. Balke, ACS Nano 5, 5683 (2011).

${ }^{37}$ P. S. Zelenovskiy, V. Ya. Shur, P. Bourson, M. D. Fontana, D. K. Kuznetsov, and E. A. Mingaliev, Ferroelectrics 398, 34 (2010).

${ }^{38}$ V. Ya. Shur, V. A. Shikhova, A. V. Ievlev, P. S. Zelenovskiy, M. M. Neradovskiy, D. V. Pelegov, and L. I. Ivleva, J. Appl. Phys. 112, 064117 (2012).

${ }^{39}$ E. A. Eliseev, A. N. Morozovska, G. S. Svechnikov, V. Gopalan, and V. Ya. Shur, Phys. Rev. B 83, 235313 (2011).

${ }^{40}$ M. Schröder, A. Haußmann, A. Thiessen, E. Soergel, T. Woike, and L. M. Eng, Adv. Funct. Mater. 22, 3936 (2012).
${ }^{41}$ W. Ge, H. Zeng, Y. Shuai, and W. Zhang, Mater. Res. Express 6, 035033 (2018).

${ }^{42}$ A. V. Ievlev, S. Jesse, A. N. Morozovska, E. Strelcov, E. A. Eliseev, Y. V. Pershin, A. Kumar, V. Y. Shur, and S. V. Kalinin, Nat. Phys. 10, 59 (2014).

${ }^{43}$ A. K. Tagantsev, I. Stolichnov, E. L. Colla, and N. Setter, J. Appl. Phys. 90, 1387 (2001).

${ }^{44}$ V. Ya. Shur, A. L. Gruverman, V. V. Letuchev, E. L. Rumyantsev, and A. L. Subbotin, Ferroelectrics 98, 29 (1989).

${ }^{45}$ V. Ya. Shur, A. L. Gruverman, N. Yu. Ponomarev, E. L. Rumyantsev, and N. A. Tonkachyova, JETP Lett. 53, 615 (1991). 